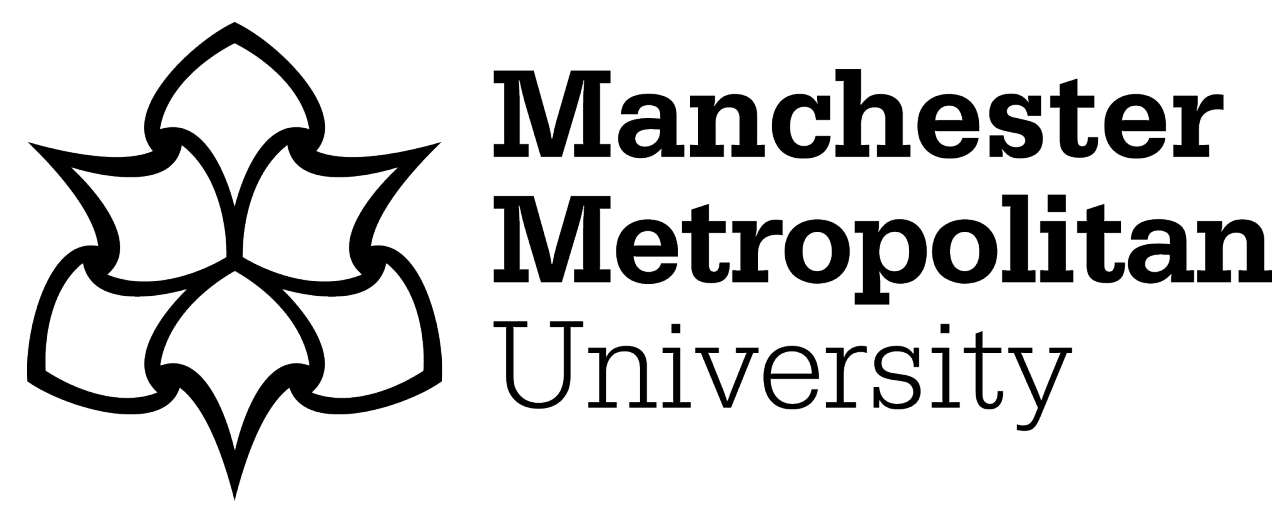

Anoh, Kelvin, Bajovic, Dragana, Vukobratovic, Dejan, Adebisi, Bamidele, Jakovetic, Dusan and Cosovic, Mirsad (2018) Distributed Energy Trading with Communication Constraints. In: 8th IEEE PES Innovative Smart Grid Technologies Conference Europe, 21 October 2018 - 25 October 2018, Sarajevo, Bosnia and Herzegovina.

Downloaded from: https: //e-space.mmu.ac.uk/621783/

Version: Accepted Version

DOI: https://doi.org/10.1109/ISGTEurope.2018.8571810

Please cite the published version 


\title{
Distributed Energy Trading with Communication Constraints
}

\author{
Kelvin Anoh $^{\dagger}$, Dragana Bajovic ${ }^{\ddagger}$, Dejan Vukobratovic ${ }^{\ddagger}$, Bamidele Adebisi ${ }^{\dagger}$, Dusan Jakovetic ${ }^{\S}$ and Mirsad \\ Cosovic $\ddagger$ \\ ${ }^{\dagger}$ School of Engineering, Manchester Metropolitan University, M1 5GD, United Kingdom \\ ${ }^{\S}$ Faculty of Technical Sciences, University of Novi Sad, Serbia \\ ${ }^{\ddagger}$ Faculty of Sciences, University of Novi Sad, Serbia \\ [k.anoh, b.adebisi]@mmu.ac.uk, [dbajovic, dejanv, djakovet, m.cosovic]@uns.ac.rs
}

\begin{abstract}
Distributed energy trading among energy prosumers (i.e., energy producers that also consume energy) is expected to bring significant cost benefits for the participating actors. In terms of the system architecture, physical grouping into microgrids (MG) can be further enhanced by communication infrastructure that provides support for flexible organization of prosumers into virtual MGs. However, how to manage prosumers using communication infrastructure is not widely investigated. In this paper, we propose a virtual MG architecture induced by communication constraints and consider its impact on total costs of energy trading. More precisely, we refine the distributed energy trading model considered in the recent literature with additional communication constraints and investigate impact of the resulting virtualized MG architecture on the overall energy trading costs. We show by simulations that there indeed exists an optimal energy trading architecture that achieves minimum possible energy trading cost, for any given model parameters.
\end{abstract}

Index Terms-Distributed energy trading, distributed energy generation, smart grid communications, energy cost optimization, virtual microgrid.

\section{INTRODUCTION}

Communication technology plays a critical role in the process of transition towards smart grids [1]. In recent decades, conventional power networks are becoming increasingly interconnected with different communication technologies that aim supporting various future smart grid services [2]. These communication technologies range from power-line communications and mobile cellular network technology to empower advanced metering infrastructure, to fiber-based core network technologies providing support for future smart grid services such as real-time monitoring, security and control [3]-[6].

Smart grids evolved into resourcing electrical energy generation from different renewable sources such as wind, water and solar power. Among these generators, an increasing number represents distributed energy generators that may both produce as well as consume energy which are referred to as prosumers [7], [8]. Besides generating and consuming, prosumers can trade energy and in the process, each prosumer pursues to maximize its own energy trading gains. However, to achieve energy trading gains, large collections of prosumers need to be studied for the best architecture that facilitates their efficient management. This leads to recent interest in the design, modeling and implementation of peer-to-peer (P2P) energy trading systems, where prosumers represent peers of the energy trading process [9].

In the literature, it has been demonstrated that prosumers are best managed using $\mathrm{P} 2 \mathrm{P}$ algorithms [8]. For a large area, the random-like nature of the distribution of prosumers may result in different challenges during energy trading. Some of these challenges include network congestion, network inefficiencies, high energy cost, security, etc. [10]. To solve these problems, large area of distributed energy prosumers can be divided into groups to enhance management, increase energy efficiency and reduce energy cost, such as in microgrids [11]. Energy trading process among such groups of prosumers spread over large geographical areas may be formulated as a distributed energy trading problem and addressed using tools from optimization or game theory, as recently investigated in [12] [13].

In this study, we propose a communication-constrained architecture that allows logical grouping of prosumers into virtual microgrids instead of physical microgrids. We assume each prosumer is equipped with energy trading client (ETC) that uses wireless technology to connect to energy trading system (ETS). Energy trading system is decentralized and comprises a large number of energy trading agents (ETA) ${ }^{1}$. The communication constraints in this context define boundaries within which ETCs are allowed to trade coordinated by their local ETAs. We note that this model is well aligned with emerging 5G mobile cellular networks that provide machinetype communication (MTC) services for ETC wireless connectivity, and mobile edge computing (MEC) architecture that supports storage and computation requirements of ETAs [5]. Localized energy trading is meaningful both in power and communication infrastructure context. Indeed, due to energy transfer costs that grow with distance between trading peers, energy trading in local communities is encouraged. Similarly, communicating energy transactions among local peers relieves the mobile core network from the burden of huge connection densities that large population of peers would induce if allowed to trade globally. On the other hand, confining energy trading to small communities reduces the available trading options and might increase the total energy trading costs. It is exactly this trade-off that we are investigating in this paper in the context

\footnotetext{
${ }^{1}$ An agent in this context is a software or hardware capable of interacting with other agents or customers [14] during a transaction process among prosumers.
} 
of placing additional communication-induced constraints to the energy trading model analyzed in [12].

The paper is organized as follows. In Sec. II, we provide background on energy trading model [12] and introduce the communication infrastructure model under study in this paper. Communication-constrained energy trading model is then proposed and analyzed in Sec. III. Numerical results resulting from optimized energy trading schedules in a given model are presented in Sec. IV. The paper is concluded in Sec. V.

\section{BACKGROUND}

In this section, we review the energy trading model to be built upon in our investigation. Further, we consider a communication network infrastructure for energy trading. The communication network introduces additional constraints to the energy trading model, which is the focus of this paper.

\section{A. Energy Trading Model}

Consider a large geographical area containing many independent energy prosumers: entities that both produce and consume energy that are also referred to as peers [7]. The peers may be of different sizes, ranging from individual households or electrical vehicles, to larger micro-grids such as wind farms. We assume that $N$ such peers are connected to the power distribution network, and are allowed to trade energy (e.g., the peers with surplus in energy production might want to sell energy to energy-deficient peers). Next, we provide a formal layout for such energy trading in the form of energy trading model proposed in [12].

A pair of peers is connected (in the energy trading sense) if they are allowed to trade energy. Note that the energy trading constraints might arise due to electrical and/or communication connectivity or administrative constraints. The peer connectivity may be defined using the energy trading graph $\mathcal{G}=(\mathcal{P}, \mathcal{E})$, whose set of nodes $\mathcal{P}=\left\{P_{1}, P_{2}, \ldots, P_{N}\right\}$ represents peers, while the set of edges $\mathcal{E} \subset \mathcal{P} \times \mathcal{P}$ defines energy trading connectivity. More precisely, an edge $\left(P_{i}, P_{j}\right) \in \mathcal{E}$ if $P_{j}$ is allowed to buy energy from $P_{i}$ (i.e., $P_{i}$ is allowed to sell energy to $\left.P_{j}\right)$. We denote by $\Omega(i)=\left\{j:\left(P_{j}, P_{i}\right) \in \mathcal{E}\right\}$ all peers that are allowed to sell energy to peer $P_{i}$, which we call the set of (in-)neighbors of $P_{i}$ (in $\mathcal{G}$ ); similarly, by $\Omega^{-}(i)=\left\{j:\left(P_{i}, P_{j}\right) \in \mathcal{E}\right\}$ we denote the set of all peers that are allowed to buy energy from peer $P_{i}$, which we will call the set of out-neighbors of $P_{i}$.

Equivalently to energy trading graph, the energy trading connectivity among peers may be defined via symmetric adjacency matrix $\mathbf{A}$ of graph $\mathcal{G}$, namely, $\mathbf{A}=\left[a_{i j}\right]_{N \times N}$, where $a_{i j}$ is a binary entry equal to one if $\left(P_{j}, P_{i}\right) \in \mathcal{E}$, otherwise it is equal to zero. We note that $\mathbf{A}$ may also be nonsymmetric in case that peers are permitted to transact energy in one direction only, in which case the energy trading graph $\mathcal{G}$ is directed.

We assume the time is divided into scheduling intervals. Within each scheduling interval, the peer $P_{i}$ generates $E_{i}^{(g)}$ and consumes $E_{i}^{(c)}$ units of energy. In addition, during each scheduling interval, conditioned that $\left(P_{i}, P_{j}\right) \in \mathcal{E}, P_{i}$ is allowed to sell energy to peer $P_{j}$. We denote by $E_{i j}$ the amount of energy peer $P_{i}$ sells to peer $P_{j}$. Then for each scheduling interval, and assuming that peers do not have the capacity to store energy, we summarize the energy generation and trading activities of peers $P_{i}, 1 \leq i \leq N$, via the following energy balance equation which holds for each peer $P_{i}$ :

$$
E_{i}^{(g)}+\sum_{j \in \Omega(i)} E_{j i}=E_{i}^{(c)}+\sum_{j \in \Omega^{-}(i)} E_{i j} .
$$

When $\mathcal{G}$ is symmetric, by rearranging terms in (1), the amount of energy generated by each peer $P_{i}$ can be more compactly expressed as:

$$
E_{i}^{(g)}=E_{i}^{(c)}+\sum_{j \in \Omega(i)}\left(E_{i j}-E_{j i}\right) .
$$

Furthermore, the energy trading model includes the costs that are incurred for generating and trading energy [12]. Let $C_{i}(E)$ be the cost of generating the amount of energy $E$ at peer $P_{i}$. Similarly, let the cost of trading the amount of energy $E$ from peer $P_{i}$ to peer $P_{j}$ be $\gamma_{i j}(E)$. Finally, we arrive at the following energy trading optimization problem:

$$
\begin{aligned}
& C^{*}=\min _{\left\{E_{i j}\right\}_{(i, j) \in \mathcal{E}}} \sum_{i=1}^{N} C_{i}\left(E_{i}^{(c)}+\sum_{j \in \Omega(i)}\left(E_{i j}-E_{j i}\right)\right)+\cdots \\
& +\sum_{j \in \Omega(i)} \gamma_{j i}\left(E_{j i}\right) \\
& E_{i j} \geq 0, \quad \forall(i, j):\left(P_{i}, P_{j}\right) \in \mathcal{E} \\
& E_{i}^{(c)}+\sum_{j \in \Omega(i)}\left(E_{i j}-E_{j i}\right) \geq 0, \quad \forall i
\end{aligned}
$$

The solution to the above optimization problem provides the

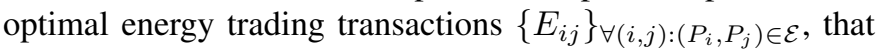
minimizes the total system costs of generation and trading, while ensuring balance equations are met.

In this work, for simplicity, we consider the case when the cost functions $C_{i}\left(E_{i}^{(g)}\right)$ and $\gamma_{i j}\left(E_{i j}\right)$ are linear. More specifically, we assume $C_{i}(E)=C_{i} \cdot E$ and $\gamma_{i j}(E)=\gamma_{i j} \cdot E$, where $C_{i}$ and $\gamma_{i j}$ are unit-costs of generating and trading energy, respectively.

\section{B. Communication Infrastructure Model}

Energy trading model in the previous subsection relies on both power and communication infrastructure. As much as the power infrastructure is needed to transfer the traded energy, the communication infrastructure is instrumental for energy transactions that dictate the energy trading process.

For the communication infrastructure model, we assume each peer participates in energy trading process via its ETC. The ETC at peer $P_{i}$ provides the energy trading system with estimates of generated and consumed energy in the upcoming scheduling interval, as well as the generation and energy transmission unit-costs. ETC is placed at the peer location and contains an integrated communication module with an interface towards an external access network for data connectivity with the rest of the energy trading system.

On the infrastructure side, the energy trading system consists of one or more ETAs. Prior to each scheduling interval, ETAs process inputs from all ETCs, and provide optimal energy trading transactions $\left\{E_{i j}\right\}$ back to all ETCs. We consider 


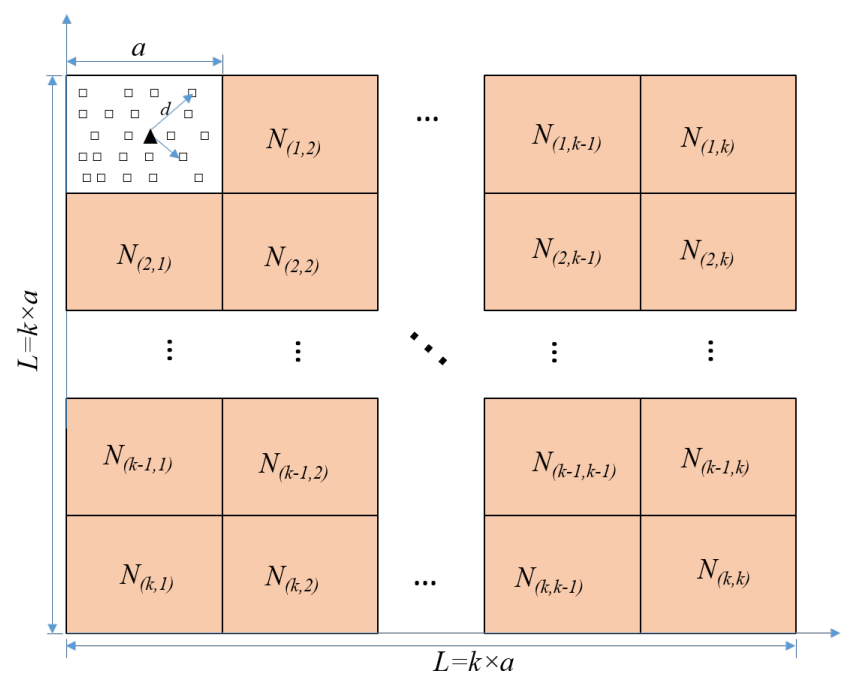

(a) A large trading area split up by ETA into squares each with length $a$.

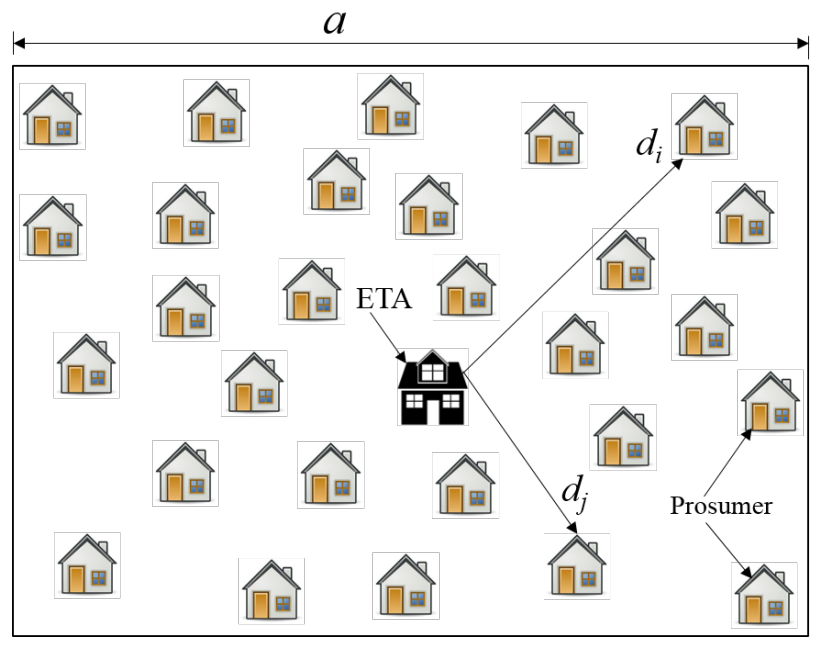

(b) An enlarged ETA trading area

Figure 1: A large ETS area divided into $M$ unit areas by an ETA. The peers are constrained within the reach of an ETA. We denote by $d_{i}$ the Euclidean distance between prosumer $i$ and the respective ETA.

three different architectures for the overall ETS depending on the number $M$ of ETAs:

Centralized ETS $(M=1)$ : The centralized architecture considers a single ETA located at the central computation node (e.g., the cloud) which processes inputs from all ETCs in the system and solves the energy trading optimization problem centrally.

Distributed Multi-Area ETS $(M<N)$ : The distributed multi-area architecture assumes a large number of geographically distributed ETAs located at network-edge computation nodes (e.g., the fog/edge). ETAs process inputs from local ETCs and communicate with neighboring ETAs in order to provide the solution to the energy trading optimization problem using distributed optimization techniques.

Fully Distributed ETS $(M=N)$ : The extreme version of the distributed multi-area ETS is the case in which each peer contains both ETS and ETA. Thus ETAs are no longer located in core network, but only at the end user (i.e., peer) location. Distributed optimization algorithms are also applied to solve the energy trading optimization problem.

In this work, we are interested in the energy trading model for a scenario with massive number of peers $N$. For this reason, our focus is on the distributed multi-area ETS, and in particular, on investigation of the energy trading model for different $M / N$-ratios (i.e., different area sizes). Indeed, for large $N$, centralized ETS suffers from huge communication burden, further limited by large communication delays and potential complexity issues related to solving very large energy trading optimization problem centrally. In contrast, at the opposite end, fully distributed ETS that applies distributed optimization across very large number of agents might suffer from slow convergence and large communication overhead, deeming them impractical in real-world applications.

\section{COMmunication-Constrained EnERgy Trading MODEL}

We model the total ETS area as a unit square. Over this area, $M=k^{2}$ ETAs are deployed in a regular fashion. This induces the division of the ETS area into $M$ smaller square regions each of which is of width $a=1 / \sqrt{M}$, as shown in Fig. 1, where each ETA is responsible for handling energy transactions of peers located in the respective region. Letting $m$ index the ETAs and ETA areas (the labeling can be performed, e.g., in a "row by row" fashion), we denote by $N_{m}$ the number of peers located in the $m$-th region, $m=1,2, \ldots, M$.

As discussed in Section II, when the number of peers $N$ is very large (e.g., $N$ is the number of households and/or electric vehicles in a given country), optimizing the energy transactions $\left\{E_{i j}\right\}_{\forall(i, j):\left(P_{i}, P_{j}\right) \in \mathcal{E}}$ at the system-wide level might not be possible to achieve in real time. Thus, we propose an architecture where energy trading is localized to the ETA areas such that two nodes can trade energy only if they belong to the same ETA area. We additionally assume that any two nodes belonging to the same area are allowed to trade energy. In terms of topology, the graph of the described ETS consists of $M$ components, each of which is a complete graph on $n_{m}$ nodes, where $m$ is the index of the corresponding ETA area. It is easy to show that the associated adjacency matrix then has the following block-diagonal form $\mathbf{A}=\operatorname{diag}\left(1_{n_{1}} 1_{n_{1}}^{\top}-I_{n_{1}}, \ldots, 1_{n_{M}} 1_{n_{M}}^{\top}-\mathbf{I}_{n_{M}}\right)$, where $1_{l}$ and $\mathbf{I}_{l}$ are, respectively, the vector of all ones and the identity matrix of size $l$, where $l$ is an arbitrary integer.

Given the above ETS description, the cost of energy trading within area $m$ is given by

$\mathcal{C}_{m}=\sum_{i=1}^{N_{m}} C_{i, m}\left(E_{i, m}^{(c)}+\sum_{j=1}^{n_{m}}\left(E_{i j, m}-E_{j i, m}\right)\right)+\sum_{j=1}^{N_{m}} \gamma_{j i, m} E_{j i, m}$ 
To analyze the preceding cost, we consider a fixed ETA area, and, to simplify the exposition, we suppress the corresponding index $m$ in all the quantities in (4) where this index occurs. Let $E_{i i}$ denote the amount of energy produced at peer $i$ to be used locally, i.e., for own consumption. It is easy to see that we can then write

$$
E_{i}^{(g)}=E_{i i}+\sum_{j=1}^{n} E_{i j}, \quad E_{i}^{(c)}=E_{i i}+\sum_{j=1}^{n} E_{j i} .
$$

In other words, the left hand-side identity in (5) is simply saying that the amount of energy generated at node $i$ is the sum of the amount of energy produced for local usage and the amount of energy that $i$ sells to other peers. Similar interpretation holds for the right hand-side identity in (5), which factors energy consumption at $i$.

For each peer $i$, we introduce a vector $\alpha_{i} \in \mathbb{R}^{n}$, whose $j$-th entry defines which fraction of $E_{i}^{(c)}$ will be sought from peer $j$, where $j=1, \ldots, n$. Thus, $\alpha_{i j}$ 's are nonnegative numbers that sum up to 1 , i.e., $\alpha_{i j} \geq 0$, for all $j$, and $\sum_{j=1}^{n} \alpha_{i j}=1$. We call $\alpha_{i}$ the strategy of peer $i$.

Denote by $\mathcal{C}_{g}$ and $\mathcal{C}_{t}$ parts of the total cost in (4) due to energy generation and due to energy trading, respectively. Using (5), we obtain for the cost of energy generation:

$$
\begin{aligned}
\mathcal{C}_{g} & =\sum_{i=1}^{n} C_{i}\left(E_{i i}+\sum_{j=1}^{n} E_{i j}\right)=\sum_{i=1}^{n} C_{i} E_{i i}+\sum_{j=1}^{n} C_{j} E_{j i} \\
& =\sum_{i=1}^{n} \sum_{j=1}^{n} C_{j} \alpha_{i j} E_{i}^{(c)}
\end{aligned}
$$

The cost of energy trading can be similarly expressed as

$$
\mathcal{C}_{t}=\sum_{i=1}^{n} \sum_{j=1}^{n} \gamma_{j i} \alpha_{i j} E_{i}^{(c)},
$$

where we assume that $\gamma_{i i}=0$ for each $i$. From the preceding two equations we can see that, given the estimated amounts of energy $E_{i}^{(c)}$ to be consumed by the peers in the next time interval, the total energy trading cost is fully described by the peers' strategies $\alpha_{i}$. Going back to the energy trading optimization (3), we thus have

$$
\begin{array}{cc}
C^{*}=\min _{\left\{\alpha_{i}\right\}_{\forall i}} & \sum_{i=1}^{n} \sum_{j=1}^{n}\left(C_{j}+\gamma_{j i}\right) \alpha_{i j} E_{i}^{(c)} \\
\text { subject to } & \alpha_{i j} \geq 0, \sum_{j=1}^{n} \alpha_{i j}=1 \forall i, j \\
\alpha_{i} \in \mathbb{R}^{n}, \forall i .
\end{array} .
$$

Note now that the peers' strategies are decoupled, i.e., each peer can pick its own strategy independently of other peers. Furthermore, the cost function in (8) is linear and hence decomposable. Therefore, the energy trading optimization problem decomposes into $n$ individual subproblems, where, in the $i$-th such subproblem, peer $i$ finds its optimal strategy $\alpha_{i}$ by solving, for any given $E_{i}^{(c)}$,

$$
\begin{array}{lc}
C_{i}^{*}=\min _{\alpha_{i}} & \sum_{j=1}^{n}\left(C_{j}+\gamma_{j i}\right) \alpha_{i j} E_{i}^{(c)} \\
\text { subject to } & \alpha_{i j} \geq 0, \sum_{j=1}^{n} \alpha_{i j}=1, \forall j . \\
\alpha_{i} \in \mathbb{R}^{n}
\end{array}
$$

The preceding problem is a linear program. It is easy to show that its solution is the canonical vector $\alpha_{i}^{\star}=e_{j^{\star}}$, where

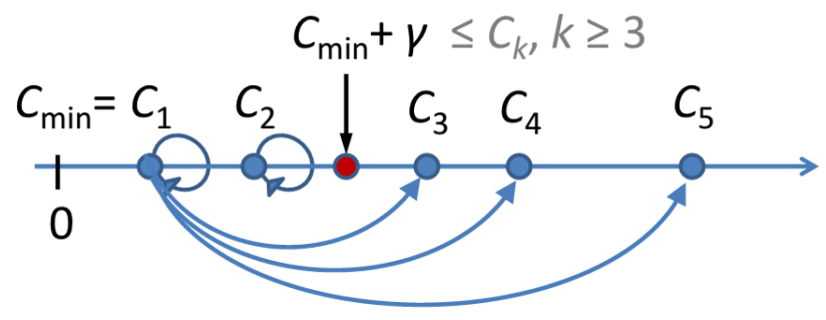

Figure 2: Illustration of the optimal energy trading strategies for a 5 prosumer network; the energy generation costs satisfy $C_{\min }=C_{1} \leq C_{2} \leq \cdots \leq C_{5}$ and the energy transmission cost $\gamma$ is such that $C_{2}<C_{\min }+\gamma<C_{3}$. Optimal trading strategies for each prosumer are represented by directed links and self-loops.

$j^{\star}=\arg \min _{j=1, \ldots, n} C_{j}+\gamma_{i j}$, with the optimal value equal to $\mathcal{C}_{i}^{\star}=\left(C_{j^{\star}}+\gamma_{j^{\star} i}\right)$. If for every $j \neq i, C_{i} \leq C_{j}+\gamma_{j i}$, then $j^{\star}=i$. Otherwise, $j^{\star}$ is the peer for which $C_{j}+\gamma_{j i}$ is minimal. Summarizing, each peer will either generate by itself all energy it needs to consume, or it will buy all the energy it needs from a single peer - the one whose sum of the per-unit generation and transmission cost is the lowest. We note that this single-point of trading form of the optimal strategy is a consequence of the fact that the energy generation and energy trading costs are assumed to be linear. When the costs are nonlinear (e.g., quadratic) this will no longer be the case [12]; we leave analysis of such a case with additional communication constraints for our future work.

Special case: $\gamma_{i j} \equiv \gamma$. In the special case when all energy transactions occurring in the same ETA area have equal perunit transmission costs, $\gamma_{i j} \equiv \gamma$, for $i \neq j$, the peers' optimal local strategies $\alpha_{i}^{\star}$ exhibit an interesting structure. To explain this, let $C_{\min }=\min \left\{C_{i}: i=1, \ldots, n\right\}$ be the minimal cost of generating one unit of energy across the entire ETA area. For any given peer $i$, it can easily be shown that the problem (9) then simplifies to finding the minimum $\min \left\{C_{i}, C_{\text {min }}+\gamma\right\}$. Therefore, if the cost of generation and transmission of the cheapest peer is smaller than its local energy production cost, node $i$ will buy all the energy it needs from the cheapest peer (assuming the cheapest peer can produce the demanded energy). Otherwise, it will produce the needed energy locally. The overall energy trading cost (8) in this case therefore equals:

$$
\mathcal{C}^{\star}=\sum_{i=1}^{n} C_{i} \mathbf{1}_{\left\{C_{i} \leq C_{\min }+\gamma\right\}}+\sum_{i=1}^{n}\left(C_{\min }+\gamma\right) \mathbf{1}_{\left\{C_{i}>C_{\min }+\gamma\right\}},
$$

where $1_{A}$ is the indicator function of event $A$.

The form of the optimal strategies is illustrated in Fig. 2 for a network consisting of 5 prosumers (and, as described earlier, where each pair of prosumers is allowed to trade). Labeling the prosumers in the increasing order according to their energy generation costs, we have $C_{\min }=C_{1} \leq C_{2} \leq$ $\ldots \leq C_{5}$. The energy transmission cost $\gamma$, equal for all pairs of prosumers, is such that $C_{2}<C_{\min }+\gamma<C_{3}$. It can be seen that for prosumers 1 and 2 their local energy generation 


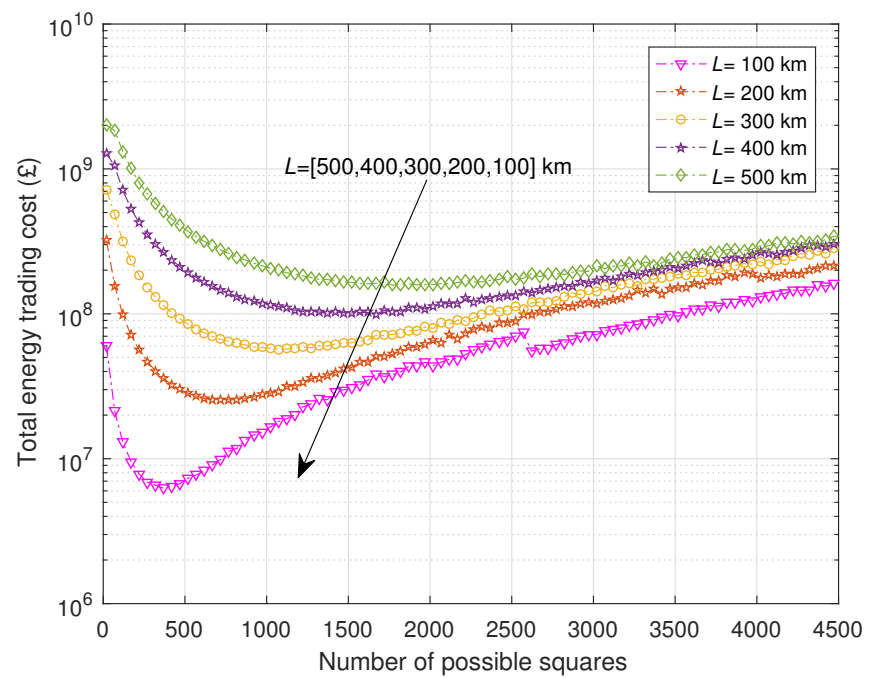

Figure 3: Total energy production costs for different large areas divided into small areas using ETAs

costs are lower than the cost of buying and transmitting energy from the cheapest producer. Thus, the corresponding optimal strategies are to produce all the needed energy locally, which is indicated by the self-loops around points $C_{1}$ and $C_{2}$ denoting prosumers 1 and 2 in Fig. 2. On the other hand, for prosumers 3,4 and 5, their local energy generation costs are still higher than the cost of both transmission and energy generation at the cheapest producer - prosumer 1 . Hence these prosumers will buy all their respective energies from prosumer 1; the directed links in Fig. 2 originating at prosumer 1 indicate the respective energy flows.

Optimizing the number of ETA areas $M$. In this paper, our primary interest is to explore the tradeoffs incurred by the size $a^{2}=1 / M$ of one ETA region, or, equivalently, by the number of divisions $M$ of a given ETS with $N$ prosumers. It is clear that when $M$ grows large, each of the areas gets smaller, and hence we can expect that the energy trasmission costs $\gamma_{i j}$ decrease. On the other hand, as the total number of prosumers stays constant, the per-area number of prosumers $n_{m}$ gets smaller. In a sense, the market offer/diversity in terms of energy generation cost then reduces. In terms of the model described above, the cost of the cheapest producer $C_{\min }$ for each of the areas then increases, and hence one can expect that the power generation component of the trading cost increases. These interplays between the two costs for each of the areas are then scaled up to the total ETS system, resulting in a highly non-trivial dependence of the total cost $\mathcal{C}^{\star}$ on $M$. In Section 4 we show by simulations that there exists an optimal ETS division which has a minimal cost among all possible divisions of size $M=k^{2}$, where $k$ varies from one to an arbitrary large number.

\section{Numerical Results}

From the foregoing discussion, we model an exemplar large area of length, $L=500 \mathrm{~km}$ or measuring $250,000 \mathrm{~km}^{2}$ in

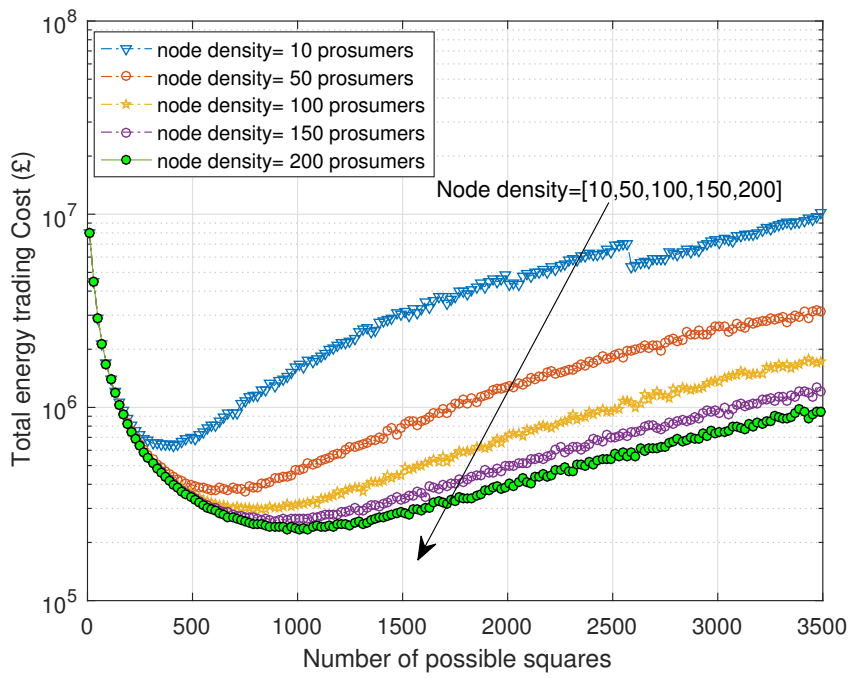

Figure 4: Normalized energy trading cost performances for varying number of prosumers in each ETA cluster-division of a large area measuring $100 \times 100 \mathrm{~km}^{2}$

area. Then, we seek to find the best number of ETAs that can be deployed in the area to achieve minimum cost of energy trading. In addition, we further assume that energy generation costs are drawn according to the uniform distribution $[0,16]$ pence per unit $(\mathrm{kWh})$ energy. We assume that there are 10 prosumers per smallest possible area (corresponding to the maximal $\left.k_{\max }=5000\right)$, resulting in total of $250 \times 10^{6}$ prosumers across entire ETS.

For the energy transmission cost we assume that it increases linearly with the size $a$ of ETA square; more specifically, we let $\gamma(k)=10 \times k_{\max } / k$, where the number of divisions $M=$ $k^{2}$ (the unit energy transmission cost for the smallest possible ETA area then equals 10 pence). Using the optimal model in (10), we present the optimal number of ETAs that can be introduced in the large area to attain minimal cost as shown in Fig. 3. It can be observed that the division of the large area attains a minimum cost $\left(£ 1.2955 \times 10^{6}\right)$ when it is divided into 1620; in other words there are 1620 ETAs required to achieve minimum energy cost. Afterwards, dividing the area into any further smaller unit squares again increases the cost. Additionally, we simulated the scenario for other areas, namely $(400,300,200$ and 100 $) \times 10^{3} \mathrm{~km}^{2}$ respectively. The results follow similar trend as in the $500 \times 10^{3} \mathrm{~km}^{2}$ area case. For example, the minimum costs for $(400,300,200$ and 100$) \times 10^{3}$ $\mathrm{km}^{2}$ areas can be observed as $\left(£ 0.8283 \times 10^{6}\right),(£ 0.4723 \times$ $\left.10^{6}\right),\left(£ 0.2083 \times 10^{6}\right)$ and $\left(£ 0.0519 \times 10^{6}\right)$, with optimal divisions into, respectively, 1270, 870, 670 and 370 ETA areas.

Further, consider the area of $100 \times 100 \mathrm{~km}^{2}$, and vary the density of users by varying the number of users $n_{0}$ in the smallest possible ETA, $n_{0}=10,50,100,150,200$. The results are shown in Fig. 4, where we note that, for each different $n_{0}$, and at each point $k$, the obtained cost is normalized by the corresponding total number of prosumers. The results show that the (per-prosumer) cost decreases when the node density increases. From the theoretical perspective, lower node 


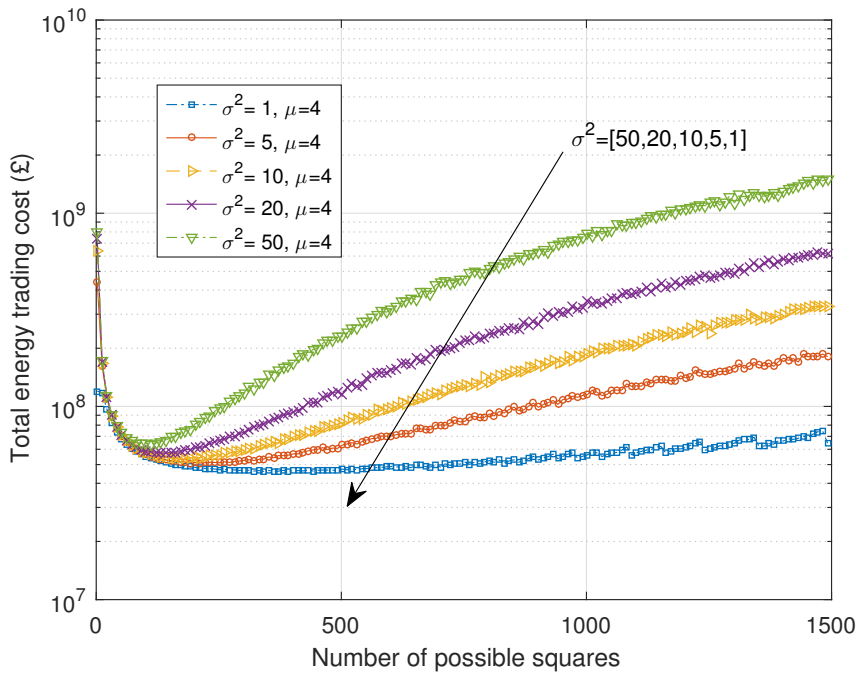

Figure 5: Effect of model distribution variance on energy trading cost over an area $100 \times 100 \mathrm{~km}^{2}$

density means higher values of $C_{\min }$ (i.e., more expensive cheapest producer), which then results in the increased energy generation and hence overall energy trading costs. On the more practical side, the theoretical predictions are very well aligned with the intuition that it is really more expensive to trade with fewer prosumers in a microgrid, compared to the case when the market has a more diverse offer.

In the final set of simulations, we investigate effects of varying parameters of the energy generation cost distribution. Specifically, we generate energy costs according to the uniform distribution in the interval $\mu+\sigma \times[1,16]$. For the results shown in Fig. 5 we set $\mu=4$ and we vary the spread of the costs by varying $\sigma^{2}=1,5,10,15,20$. By increasing $\sigma$ we effectively decrease the ratio between the energy transmission cost $\gamma$ and the average energy generation cost (which with $\sigma$ increase according to $\mu+\sigma \times 8$ pence).

One can observe from the obtained curves that the optimal division slightly shifts to lower values of $k$ as $\sigma$ increases. The intuition behind this effect is that, when increasing $\sigma$, the average energy generation cost increases relative to the unit distance energy transmission cost. Thus, there will be more peers buying the energy from the cheapest prosumer, rather than producing the energy locally. Hence, the determining factor in the energy trading cost becomes the value $C_{\min }$. As the size of the area increases, $C_{\min }$ gets smaller and hence, with larger values of $\sigma$ the system tends to pick larger squares to compensate with smaller $C_{\min }$ for the increasing average energy generation cost.

\section{CONCLUSION}

Motivated by the increasing scale of the envisioned energy trading systems, we propose a distributed energy trading system where the trading topology is dictated by the underlying communication architecture. Grouping the prosumers to their closest trading agents, we ask the question what is the optimal group size which results in the minimal overall trading cost.
We show by simulations for various problem parameters that there indeed exists a non-trivial system division. The respective prosumer clustering results in the lowest energy trading cost, among all possible system divisions - ranging from the full-scale trading to the arbitrary small clusters (e.g., on the size of a couple of households). As a future work, we plan to address the described optimal trading architecture problem analytically, as well as with different energy generation and transmission cost models (e.g., quadratic, or general convex functions).

\section{REFERENCES}

[1] H. Farhangi. The path of the smart grid. IEEE Power Energy Mag., 8(1), 2010.

[2] Y. Yan, Y. Qian, H. Sharif, and D. Tipper. A survey on smart grid communication infrastructures: Motivations, requirements and challenges. IEEE Commun. Surveys Tut., 15(1):5-20, 2013.

[3] S. Galli, A. Scaglione, and Z. Wang. For the grid and through the grid: The role of power line communications in the smart grid. Proceed. IEEE, 99(6):998-1027, 2011.

[4] A. Ikpehai, B. Adebisi, and K. Rabie. Broadband PLC for clustered advanced metering infrastructure (AMI) architecture. Energies, 9(7):569, 2016.

[5] M. Cosovic, A. Tsitsimelis, D. Vukobratovic, J. Matamoros, and C. Anton Haro. 5G mobile cellular networks: Enabling distributed state estimation for smart grids. IEEE commun. Mag., 55(10):62-69, 2017.

[6] S. M. Amin and B. F. Wollenberg. Toward a smart grid: power delivery for the 21st century. IEEE Power Energy Mag., 3(5):34-41, 2005.

[7] C. Marnay, B. Nordman, and J. Lai. Future roles of milli-, micro-, and nano-grids. In CIGRE Intl. Symposium The electric power syst. futureIntegrating supergrids and microgrids, Bologna, Italy., Sept. 2012.

[8] H. Almasalma, J. Engels, and G. Deconinck. Peer-to-peer control of microgrids. arXiv preprint arXiv:1711.04070, 2017.

[9] O. Jogunola, A. Ikpehai, K. Anoh, B. Adebisi, M. Hammoudeh, S.Y. Son, and G. Harris. State-of-the-art and prospects for peer-to-peer transaction-based energy system. Energies, 10(12):1-28, Dec. 2017.

[10] A. Bari, J. Jiang, W. Saad, and A. Jaekel. Challenges in the smart grid applications: an overview. Int. J. Distributed Sensor Netw., 2014, 2014.

[11] R. H. Lasseter and P. Paigi. Microgrid: a conceptual solution. In IEEE 35th Annual Power Electron. Specialists Conf., volume 6, pages 42854290 Vol.6, Jun. 2004.

[12] D. Gregoratti and J. Matamoros. Distributed Energy Trading: The Multiple-Microgrid Case. IEEE Trans. Ind. Electron., 62(4):2551-2559, Apr. 2015.

[13] Y. Wang, W. Saad, Z. Han, H. V. Poor, and T. Başar. A game-theoretic approach to energy trading in the smart grid. IEEE Trans. Smart Grid, 5(3):1439-1450, 2014.

[14] S. Suryanarayanan, J. Mitra, and S. Biswas. A conceptual framework of a hierarchically networked agent-based microgrid architecture. In IEEE PES T D 2010, pages 1-5, Apr. 2010. 